\title{
A Neural Network for Spatial and Temporal Modeling of foF2 Data Based on Satellite Measurements
}

\author{
Haris Haralambous and Harris Papadopoulos \\ Computer Science and Engineering Department, Frederick University, \\ 7 Y. Frederickou St., Palouriotisa, Nicosia 1036, Cyprus \\ $\{$ H. Haralambous, H. Papadopoulos\}@frederick.ac.cy
}

\begin{abstract}
This paper presents the application of Neural Networks for the spatial and temporal modeling of (critical frequency) foF 2 data over Europe. foF 2 is the most important parameter in describing the electron density profile of the ionosphere since it represents the critical point of maximum electron density in the profile and therefore can be used to drive empirical models of electron density which incorporate $f o F 2$ as an anchor point in the profile shape. The model is based on radio occultation (RO) measurements by LEO (Low Earth Orbit) satellites which provide excellent spatial coverage of $f o F 2$ measurements.
\end{abstract}

Keywords: Ionosphere, radio occultation, F2 layer critical frequency.

\section{Introduction}

The ionosphere is defined as a region of the earth's upper atmosphere where sufficient ionisation can exist to affect radio waves in the frequency range 1 to $3 \mathrm{GHz}$. It ranges in height above the surface of the earth from approximately $50 \mathrm{~km}$ to $1000 \mathrm{~km}$. The influence of this region on radio waves is accredited to the presence of free electrons.

The impact of the ionosphere on communication, navigation, positioning and surveillance systems is determined by variations in its electron density profile and subsequent electron content along the signal propagation path [1]. As a result satellite systems for communication and navigation, surveillance and control that are based on trans-ionospheric propagation may be affected by complex variations in the ionospheric structure in space and time leading to degradation of the accuracy, reliability and availability of their service.

The uppermost layer of the ionosphere is the F2 region which is the principal ionospheric region where electron density maximises and therefore introduces significant effects in transionospheric signals (navigation and communication) that penetrate the ionosphere. The maximum frequency that can be reflected at vertical incidence by this layer is termed the F2 layer critical frequency (foF2) and is directly related to the maximum electron density of the electron density profile (see Figure 1). The F2 layer critical frequency is therefore the most important parameter in characterising the ionospheric electron density profile. The maximum electron density

(C) IFIP International Federation for Information Processing 2012 
of free electrons within the F2 layer and therefore foF2 depend upon the strength of the solar ionising radiation which is a function of time of day, season, geographical location and solar activity $[2,3,4]$.

This paper describes the development of a neural network model which describes the temporal and spatial variability of $f o F 2$ data over a significant part of Europe. The model is based on approximately 53000 LEO satellite foF2 values from RO measurements recorded from January 2007 to December 2010. This is the first attempt to develop a foF 2 model from RO satellite measurements. The significance of this model lies in its superior spatial interpolation properties due the fact that is based on foF 2 measurements of high spatial resolution as a result of the excellent geographical coverage provided by the COSMIC satellite mission. This spatial resolution is significantly higher than that provided by ionosonde measured foF 2 values which is the traditional method of probing the ionosphere and can also extend over the sea where ionosonde radars are impractical to operate for obvious reasons.

There have been other efforts for the introduction of the spatial aspect, both in the direction of long-term prediction and short-term forecasting models of foF 2 based on ground-based foF2 measurements. Lamming and Cander (1999) [5] attempted to incorporate geographical latitude and longitude as model parameters to address the interpolation capability of a monthly median foF 2 Neural Network model between ionospheric stations. over Europe. Kumluca et al. (1999) [6] also explored this idea by including more than one ionospheric station to extend the work of Altinay et al. (1997) [7]. Oyeyemi et al. (2005) [8] used data from 40 worldwide ionospheric stations spanning the period 1964-1986 for training a global Neural Network for short-term forecasting of foF2 including geographical latitude and other geographically related geophysical parameters as inputs. Oyeyemi et al. (2008) [9] extended their previous work by including ground based ionosonde data, from 84 global stations to propose a global long-term prediction Neural Network model of foF 2 .

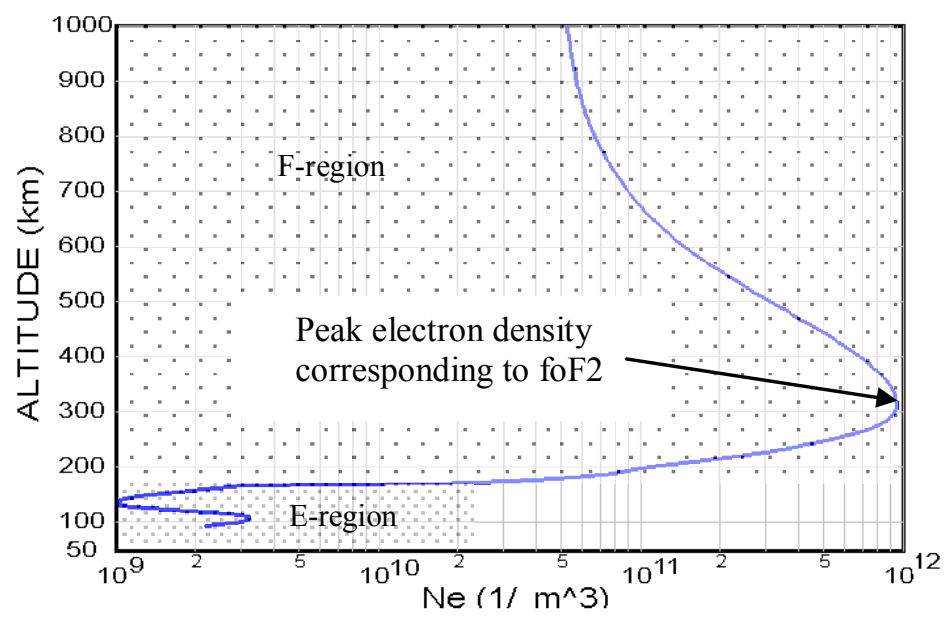

Fig. 1. Typical electron density altitude profile of the ionosphere 


\section{Measurement of F2 Layer Critical Frequency by Ground-Based and Satellite Techniques}

Traditionally measurements of $f o F 2$ were conducted by ionosondes which are special types of radar used for monitoring the electron density at various altitudes in the ionosphere up to the F2-layer peak electron density (corresponding to foF2). Their operation is based on a transmitter sweeping through the HF frequency range transmitting short pulses. These pulses are reflected at various layers of the ionosphere, and their echoes are received by the receiver giving rise to a corresponding plot of reflection altitude against frequency which is further analysed to infer the ionospheric plasma height-electron density profile (Figure 1). The maximum frequency at which an echo is received is called the critical frequency of the corresponding layer. Since the F2 layer is the most highly ionised ionosperic layer its critical frequency foF 2 is the highest frequency that can be reflected by the ionosphere.

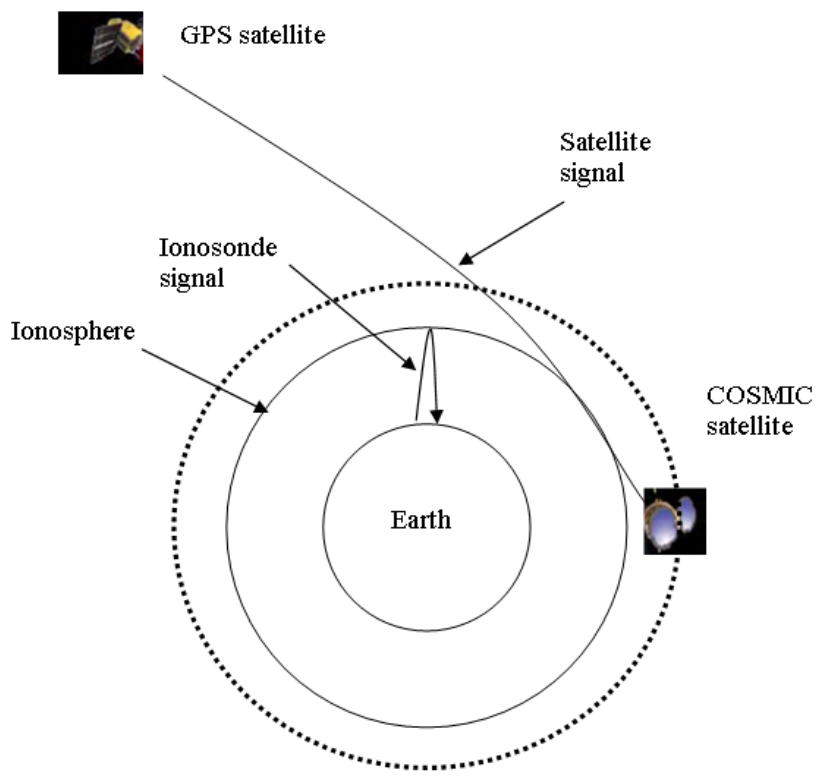

Fig. 2. Schematic illustrating a ground-based (ionosonde) and a space-based technique (satellite $\mathrm{RO})$ for probing the ionosphere

A constellation of six satellites, called the Formosa Satellite 3-Constellation Observing System for Meteorology, Ionosphere, and Climate (COSMIC), was launched on April 15, 2006 to improve the global weather prediction and space weather monitoring $[10,11]$. The instrument that is of interest in this paper is the GPS receiver which is used to obtain atmospheric and ionospheric measurements through 
phase and Doppler shifts of radio signals. The Doppler shift of the GPS L-band (L1=1575.42 MHz, L2=1227.60 MHz) signals received by a LEO satellite is used to compute the amount of signal bending that occurs as the GPS satellite sets or rises through the earth's atmosphere as seen from LEO (Figure 1). The bending angles are related to the vertical gradients of atmospheric and ionospheric refractivity which is directly proportional to ionospheric electron density above $80 \mathrm{~km}$ altitude. Through the assumption of spherical symmetry, electron density profiles can be retrieved from either the bending angles or the total electron content data (computed from the L1 and L2 phase difference) obtained from the GPS radio occultations (RO) [12].We also need to emphasise that the RO occultation technique can be applied successfully in retrieving the ionospheric electron density profile only under the assumption of spherical symmetry in the ionosphere. This assumption is not always satisfied due to significant electron density gradients that give rise to horizontal electron fluxes. This violates the requirement for electron density profile inversion producing a very unrealistic profile. In order to overcome this limitation and concentrate on good quality electron density profiles a selection process was applied in order to exclude those measurements where the distortion of the profiles was excessive. In this paper the Neural Network foF2 model is developed based on a training set of RO measurements across a part of Europe (shown by the shaded area in Figure 3) and evaluated on a test set of $f o F 2$ values obtained by European ionosondes operating in Europe (in locations shown again in Figure 3).

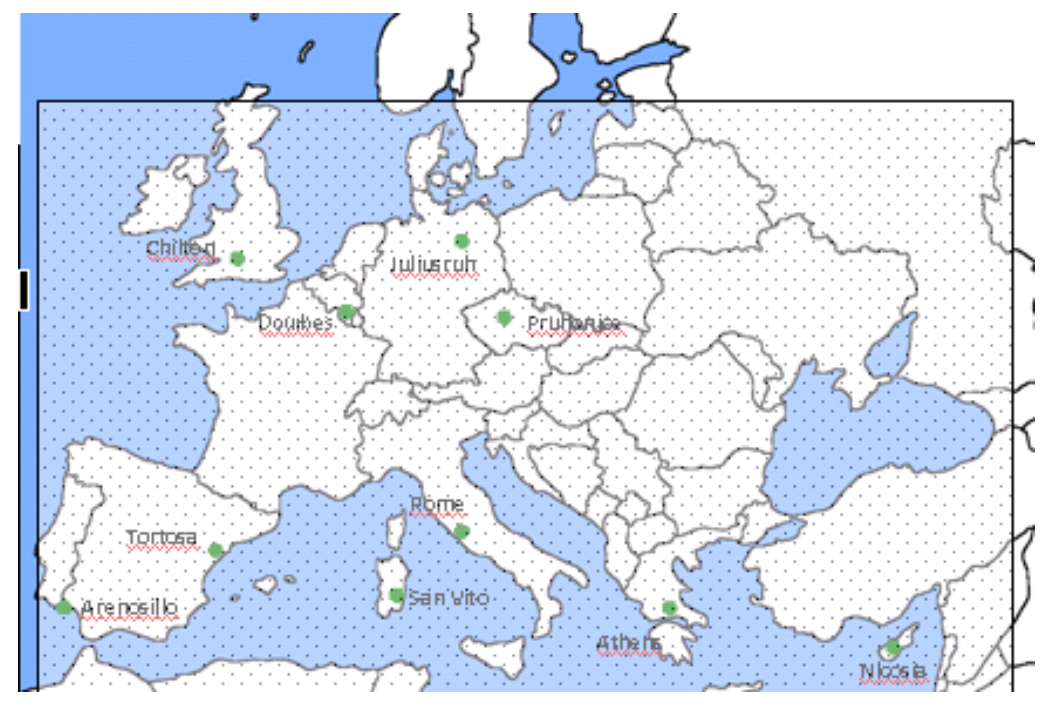

Fig. 3. A map of Europe illustrating the area within which RO measurements were considered in the model development and locations of ionosonde stations that provided the testing data sets for model evaluation 


\section{Temporal and Spatial Characteristics of the F2 Layer Critical Frequency and Model Parameters}

The temporal variability of foF 2 at a single location is well established and has been thoroughly described in previous papers $[13,14]$ primarily based on ionosonde derived foF 2 datasets. In short, ionospheric dynamics are governed principally by solar activity which in turn influences the electron density of the ionosphere. The electron density of the F2 layer exhibits variability on daily, seasonal and long-term time scales in response to the effect of solar radiation. It is also subject to abrupt variations due to enhancements of geomagnetic activity following extreme manifestations of solar activity disturbing the ionosphere from minutes to days on a local or global scale. There is also a spatial aspect of these variabilities which is depicted in Figures 4 and 6 for COSMIC foF 2 values obtained at low $\left(35^{\circ}-43^{\circ}\right)$, medium $\left(43^{\circ}-51^{\circ}\right)$ and high $\left(51^{\circ}-60^{\circ}\right)$ latitude European regions.

The most profound solar effect on $f o F 2$ is reflected on its daily variation as shown in Figure 4. As it is clearly depicted, there is a strong dependency on local time which follows a sharp increase of foF 2 around sunrise and gradual decrease around sunset. This is attributed to the rapid increase in the production of electrons due to the photoionization process during the day and a more gradual decrease due to the recombination of ions and electrons during the night. The long-term effect of solar activity on $f_{o F} 2$ follows an eleven-year cycle and is clearly shown in Figure 5 where values of foF 2 obtained from ionosonde data over Cyprus are plotted against time as well as a modeled monthly mean sunspot number $R$ which is a well established index of solar activity. We can observe a marked correlation of the mean level of foF2 and modeled sunspot number. Unfortunately using COSMIC foF 2 for such a plot was not possible as the duration of the mission coincided with an unusually extended period of low solar activity that did not allow the correlation between COSMIC foF 2 and $R$ to clearly apperar. In addition to the effects of solar activity on both parameters mentioned above we can also identify a spatial effect on the diurnal variability. The spatial aspect of temporal variability is demonstrated in Figure 4 where the diurnal variation of $f_{o F} 2$ is plotted for three different measurements corresponding to low, medium and high latitudes. It is evident from this figure that the variability is increased as latitude decreases. This spatial characteristic of diminishing foF 2 with increasing latitude is also observed in Figure 6 where the seasonal variation of the median level of foF 2 at noon recorded over the three latitude regimes over Europe (low, medium and high) is shown.

The plots in Figures 4-6 describe the variabilities that typically characterise the average temporal behaviour of foF 2 . The model parameters to describe these variabilities have been established in previous papers $[13,14]$ and are annual and daily sinusoidal components as well as a modeled sunspot number which describes the level of solar activity. In addition to these parameters latitude and longitude are introduced as additional model parameters in this paper to express the spatial variability of $f_{o F}$. 


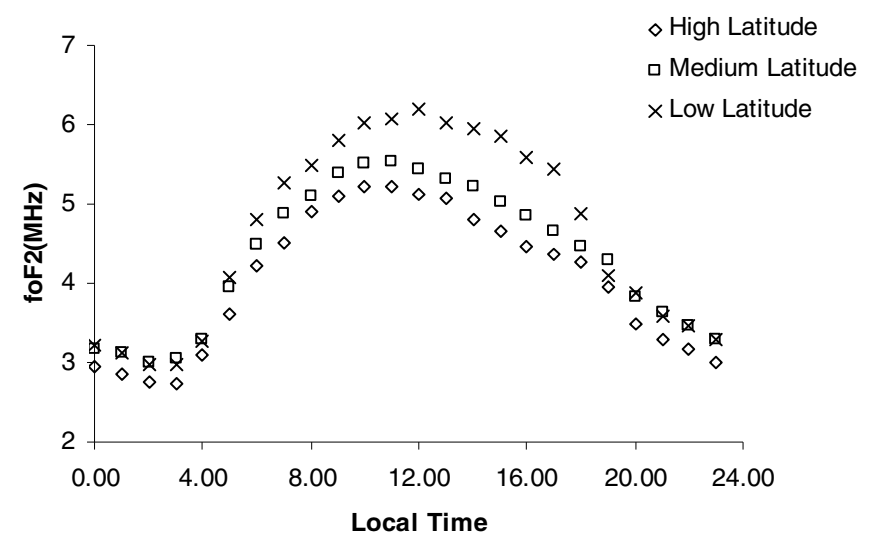

Fig. 4. Diurnal variability of foF 2 at low, medium and high latitudes
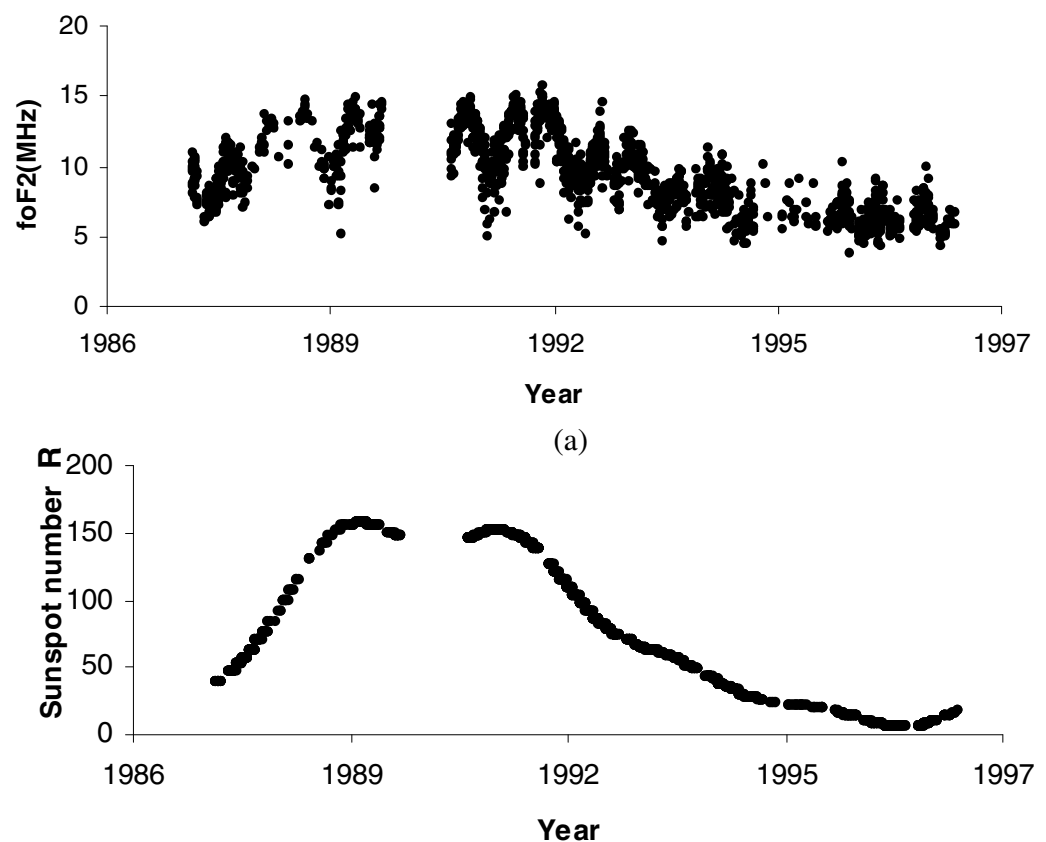

(b)

Fig. 5. Long-term foF2 and solar activity variation with time over Cyprus 


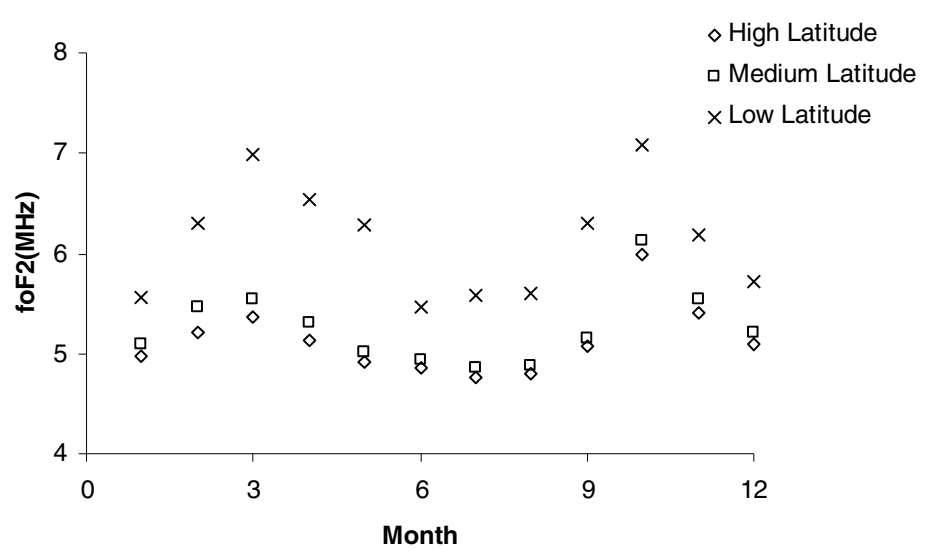

Fig. 6. Seasonal variation of $f o F 2$ at 12:00 at low, medium and high latitudes

\section{$4 \quad$ Experiments and Results}

In all experiments the available foF 2 values from RO measurements between January 2007 and May 2010 were used for training the Neural Network and values from ionosond and RO measurements between June 2010 to December 2010 were used for testing it. The Neural Networks used had a fully connected two-layer structure, with 7 input, 10 hidden and 1 output neurons. Both their hidden and output neurons consisted of hyperbolic tangent sigmoid activation functions. The number of hidden neurons was determined by trying out the values 5, 10,15 and 20. The training algorithm used was the Levenberg-Marquardt backpropagation algorithm with early stopping based on a validation set created from $20 \%$ of the training examples. In an effort to avoid local minima five NNs were trained with different random initialisations and the one that performed best on the validation set was selected for being applied to the test examples. The inputs and target outputs of the network were normalized setting their minimum value to -1 and their maximum value to 1 . This made the impact of all inputs in the model equal and transformed the target outputs to the output range of the NN activation functions. The results reported here were obtained by mapping the outputs of the network for the test examples back to their original scale.

The example plots shown in Figure 7 show the ability of the model to approximate the diurnal behaviour of $f o F 2$ at various locations over Europe. Apparently for lower latitude stations the approximation seems to be worse.

The obtained Root Mean Squared Error (RMSE) over all data for the model based on RO COSMIC measurements is shown for each site in table 1. Also RMSE values for a model based on ionosonde data and tested on COSMIC data is also given as a comparison model. 

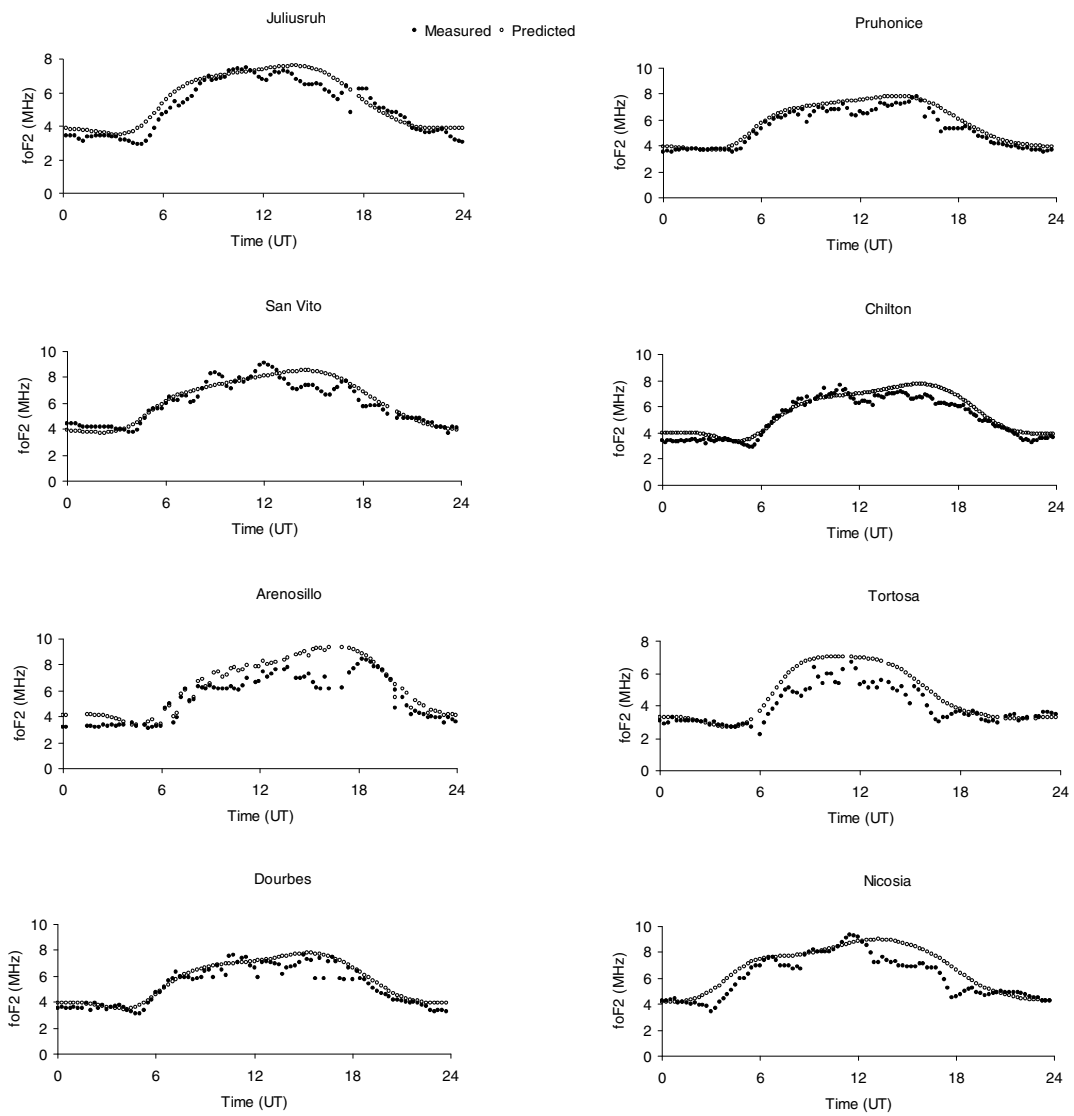

Fig. 7. Examples of measured (by ionosonde) and predicted (by COSMIC) $f o F 2$ values at various locations over Europe

A worth noting result is the plot of RMSE versus latitude given in Figure 8 for the model based on COSMIC values which clearly demonstrates the increasing trend of RMSE with latitude. This can be possibly attributed to the fact that the ability of the algorithm to infer electron density profiles may deteriorate with latitude which was a possible suggestion in the literature in the recent past [15]. Another possible reason is the increased temporal and spatial variability which characterises lower latitude regions because of the complex morphology of the ionosphere in this area. This increased variability poses an increased challenge to any effort of modeling any ionospheric characteristic such as foF 2 even under geomagnetically quiet conditions which is reflected on the higher values of RMSE. Another important point to comment on is the fact that unlike Total Electron Content (TEC -which is another very important ionospheric characteristic) values that are measured from extended networks of ground-based GPS stations sampling adequately the European sector in space and time, measurements of foF 2 from ionosondes do not achieve the same 
spatial resolution and COSMIC measurements do not achieve the same temporal resolution. Therefore techniques that have been applied in the case of interpolating TEC data are not necessarily appropriate for foF 2 modeling.

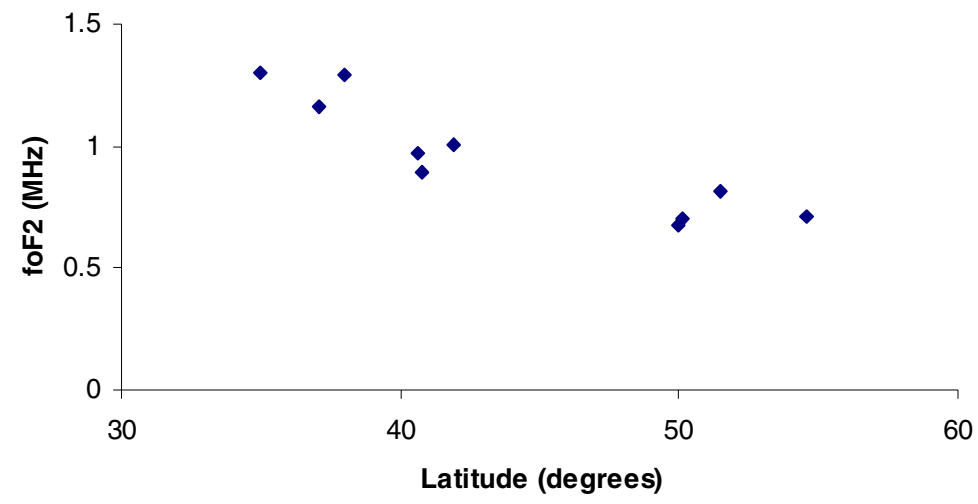

Fig. 8. RMSE versus latitude

Table 1. The Root Mean Squared Error (RMSE) of the Neural Network for the case of a COSMIC based and an ionosonde based model

\begin{tabular}{l|rc}
\hline \multirow{2}{*}{$\begin{array}{c}\text { Ionosonde } \\
\text { location }\end{array}$} & \multicolumn{2}{|c}{ RMSE (MHz) } \\
\cline { 2 - 3 } & $\begin{array}{c}\text { COSMIC } \\
\text { based }\end{array}$ & $\begin{array}{c}\text { Ionosond } \\
\text { based }\end{array}$ \\
\hline Tortosa & 0.788 & $\mathbf{0 . 7 7 9}$ \\
SanVito & $\mathbf{0 . 5 2 4}$ & 0.863 \\
Rome & $\mathbf{0 . 7 5 2}$ & 0.796 \\
Nicosia & $\mathbf{0 . 7 1 4}$ & 0.799 \\
Dourbes & 0.760 & $\mathbf{0 . 6 4 7}$ \\
Athens & $\mathbf{0 . 7 4 0}$ & 0.807 \\
Arenosillo & $\mathbf{0 . 6 6 4}$ & 1.027 \\
Pruhonic & 0.773 & $\mathbf{0 . 6 4 5}$ \\
Chilton & $\mathbf{0 . 6 6 9}$ & 0.788 \\
Juliusruh & 0.795 & $\mathbf{0 . 6 3 9}$ \\
\hline
\end{tabular}

\section{$5 \quad$ Conclusions and Future Work}

In this paper we have presented the development of a neural network tool for the spatial and temporal modeling of (critical frequency) foF 2 data over Europe. The model has been developed based on a data set obtained during a period of approximately four years from COSMIC satellite mission. The tool has produced a good interpolation capability of the gaps in the $f_{o F} 2$ data series therefore providing a 
method to preserve the variability of $f_{o F}$, a fact which is essential in the development of long-term time-series prediction models and procedures. As a next step the Neural Network approach to model foF 2 based on RO satellite measurements will be further extended to cover measurements obtained from other satellite missions (CHAMP,GRACE). The high solar activity period that is expected to reach is peak at around 2013 is expected to pose an additional challenge to such an effort. We also plan to explore the possibility of improving performance by employing more complex approaches such as an ensemble of neural networks.

\section{References}

1. Barclay, L.W.: Ionospheric Effects and Communication Systems performance. Keynote paper at the 10th Ionospheric Effects Symposium, Washington, DC (2002)

2. Goodman, J.: HF Communications, Science and Technology. Nostrand Reinhold (1992)

3. Maslin, N.: The HF Communications, a Systems Approach, San Francisco (1987)

4. McNamara, L.F.: Grid The Ionosphere: Communications, Surveillance, and Direction Finding. Krieger Publishing Company, Malabar (1991)

5. Lamming, X., Cander, L.R.: Monthly median foF2 modelling COST 251 area by neural networks. Phys. Chem. Earth (c) 24, 349-354 (1999)

6. Kumluca, A., Tulunay, E., Topalli, I., Tulunay, Y.: Temporal and spatial forecasting of ionospheric critical frequency using neural networks. Radio Sci. 34, 1497-1506 (2000)

7. Altinay, O., Tulunay, E., Tulunay, Y.: Forecasting of ionospheric critical frequency using neural networks. Geophys. Res. Lett. 24, 1467-1470 (1997)

8. Oyeyemi, E.O., Poole, A.W.V., McKinnell, L.A.: On the global model for foF2 using neural networks. Radio Sci. 40, RS6011 (2005), doi:10.1029/2004RS003223

9. Oyeyemi, E.O., McKinnell, L.A.: A new global F2 peak electron density model for the International Reference Ionosphere (IRI). Adv. Space Res. 42(4), 645-658 (2008), doi:10.1016/j.asr..10.031

10. Schreiner, W., Rocken, C., Sokolovsky, S., Syndergaard, S., Hunt, D.: Estimates of the precision of GPS radio occultations from the COSMIC/FORMOSAT-3 missio. Geophys. Res. Lett. 34, L04808 (2007), doi:10.1029/2006GL027557

11. Rocken, C., Kuo, Y.-H., Schreiner, W., Hunt, D., Sokolovsky, S., McCormick, C.: COSMIC system description. Terr. Atmos. Ocean Sci. 11, 21-52 (2000)

12. Hajj, G.A., Romans, L.J.: Ionospheric electron density profiles obtained with the Global Positioning system: Results from the GPS/MET experiment. Radio Sci. 33, 175-190 (1998)

13. Haralambous, H., Papadopoulos, H.: A Neural Network Model for the Critical Frequency of the F2 Ionospheric Layer over Cyprus. In: Palmer-Brown, D., Draganova, C., Pimenidis, E., Mouratidis, H. (eds.) EANN 2009. CCIS, vol. 43, pp. 371-377. Springer, Heidelberg (2009)

14. Haralambous, H., Ioannou, A., Papadopoulos, H.: A Neural Network Tool for the Interpolation of foF2 Data in the Presence of Sporadic E Layer. In: Iliadis, L., Jayne, C. (eds.) EANN/AIAI 2011, Part I. IFIP AICT, vol. 363, pp. 306-314. Springer, Heidelberg (2011)

15. Potula, B.S., Chu, Y.-H., Uma, G., Hsia, H.-P., Wu, K.-H.: A global comparative study on the ionospheric measurements between COSMIC radio occultation technique and IRI model. J. Geophys. Res. 116, A02310 (2011) doi:10.1029/2010JA015814 